\title{
Clinical parameters for predicting radiation- induced liver disease after intrahepatic reirradiation for hepatocellular carcinoma
}

Yaoru Huang ${ }^{1,3}$, Shang-Wen Chen ${ }^{2,3}$, Ching-Chao Fan ${ }^{4}$, Lai-Lei Ting ${ }^{1,3}$, Chia-Chun Kuo ${ }^{1,3}$ and Jeng-Fong Chiou ${ }^{1,3^{*}}$

\begin{abstract}
Background: Few data are available on the tolerance of reirradiation in patients with hepatocellular carcinoma (HCC). This study determined the clinical parameters contributing to the development of radiation-induced liver disease (RILD).

Methods: We included 36 patients with HCC who received 2 courses of radiotherapy (RT) to the liver. Using $\alpha / \beta=15$ for tumor and $\alpha / \beta=8$ for normal liver tissue for biologically equivalent doses in 2 Gy fractions, mean cumulative to the hepatic tumor and normal liver were $87.7 \mathrm{~Gy}_{15}$ and $31.1 \mathrm{~Gy}_{8}$, respectively. Hepatic toxicities were classified according to the Common Terminology Criteria for Adverse Events, Version 4.0. Clinical data, including liver function test results, radiological study findings, and RT parameters before and after both courses of RT were retrieved for analysis. Using multivariate analysis, logistic regression was used to identify the predictors of RILD, and Cox regression was performed to explore the prognostic factors for overall survival (OS).
\end{abstract}

Results: Thirteen patients (36 \%) developed RILD after reirradiation. Nine of them died because of progressive liver failure without evidence of tumor progression and were categorized to have lethal RILD. A pretreatment Child-Turcotte-Pugh (CTP) score $\geq 6$ was the only predictor of RILD [odds ratio (OR): 15.83, $p=0.001$ ] and lethal RILD [OR: 72.56, $p=0.005$ ]. In addition, a CTP score $\geq 6$ and the presence of portal vein tumor thrombosis before reirradiation were 2 prognostic factors for OS.

Conclusion: Despite a limited sample size, residual liver function using a preirradiation CTP score $\geq 6$ is a clinical parameter associated with an increased risk of RILD in patients requiring hepatic reirradiation.

Keywords: Hepatocellular carcinoma, Irradiation, Radiation-induced liver disease, Prognostic factor

\section{Introduction}

Radiotherapy (RT) has become an increasingly employed modality for treating hepatocellular carcinoma (HCC). However, few data are available on the tolerance of the liver to a second course of hepatic RT. The liver is susceptible to radiation damage; therefore, classic or nonclassic radiation-induced liver disease (RILD) has

\footnotetext{
* Correspondence: solomanc@tmu.edu.tw

'Department of Radiation Oncology, Taipei Medical University Hospital, 252, Wu Hsing St., Taipei 110, Taiwan

${ }^{3}$ Department of Radiology, School of Medicine, College of Medicine, Taipei

Medical University, Taipei, Taiwan

Full list of author information is available at the end of the article
}

been reported within weeks or months after the administration of RT [1-3]. However, the guidelines for avoiding RILD apply only to patients planning to receive the first course of hepatic RT. Whether the same guidelines are applicable to previously treated patients is unclear. In particular, $\mathrm{HCC}$ is commonly accompanied by liver cirrhosis [4-6]. Therefore, investigating hepatic tolerance when a second course of RT is required is critical.

Studies examining clinical parameters for ensuring the safety of reirradiation for recurrent hepatoma are currently lacking. Hence, to optimize patient selection, 
we conducted this study to identify risk factors associated with hepatic toxicities and treatment outcomes after reirradiation. In this study, hepatic toxicities were classified according to all RILD and lethal RILD events. Our findings can provide information about the safety and selection criteria for patients with $\mathrm{HCC}$ requiring hepatic reirradiation.

\section{Methods}

\section{Patient population}

In this study, we retrospectively analyzed the clinical data of patients who received 2 courses of RT to the liver between July 2004 and March 2014 at two hospitals. The inclusion criteria were the following: (1) a diagnosis of HCC based on tissue proof or the typical imaging appearance according to the criteria of the European Association for the Study of the Liver [7]; (2) administration of prior RT for intrahepatic HCC; (3) residual or recurrent hepatic tumors either within or outside the previous irradiation field; (4) residual or recurrent tumors being unamenable to other treatments such as surgery, transarterial chemoembolization, and radiofrequency ablation; (5) an Eastern Cooperative Oncology Group performance score of $0-1$; and (6) a Child-Pugh classification of A or B. The evaluation and treatment policies for the included patients were reviewed by a multidisciplinary team, and reirradiation was recommended.

Comprehensive clinical data of all patients were analyzed, including the age at reirradiation, the interval between the 2 courses of RT, liver function test (LFT) results, imaging study findings, and RT parameters before and after both courses of RT. The RT parameters comprised the clinical target volume (CTV), prescribed dose, normal liver reserve volume, and mean liver dose. The LFT results were the hepatitis B virus (HBV) or hepatitis C virus infection status; levels of serum alanine aminotransferase (ALT), serum aspartate aminotransferase (AST), albumin, total bilirubin, and alkaline phosphatase; prothrombin time; and Child-Turcotte-Pugh (CTP) score. In total, 36 patients were included in this study; patient characteristics are listed in Table 1 . The median age was 66 years, with a range of $32-80$ years.

\section{Target delineation and RT planning}

All patients underwent triphasic computed tomography (CT) or magnetic resonance imaging before treatment. The patients were immobilized using a vacuum bag with motion control using abdominal compression. CT simulation was performed using a 3-mm slice thickness with contrast enhancement; the simulation range included the entire liver, both lungs, and both kidneys. The gross tumor volume (GTV) was contoured as visible tumors on CT. The CTV was defined by leaving a 3-5-mm margin in three dimensions from the GTV or from the area adjacent to visible tumors where microscopic tumors were suspected. The planning target volume (PTV) was delineated as a 5-mm margin added to the CTV in three dimensions. The normal liver reserve was defined as the entire liver excluding all PTVs. According to recommended standards, the normal liver reserve was $>700 \mathrm{~mL}$, and the mean dose was restricted to $\leq 23 \mathrm{~Gy}_{2}[8,9]$. In addition, the median dose to any kidney was less than $20 \mathrm{~Gy}_{2}$, the volume of the duodenum or stomach receiving more than $50 \mathrm{~Gy}_{2}$ was restricted to $<1 \mathrm{~mL}$, the mean dose to the esophagus was less than $34 \mathrm{~Gy}_{2}$, and the cumulative doses to the spinal cord from two courses of RT was less than $60 \mathrm{~Gy}_{2}$ [10]. The same technique and similar constraints were used for both courses of RT.

All patients were treated using intensity-modulated RT (IMRT) with or without image guidance or image-guided tomotherapy. For patients treated with IMRT, static and coplanar procedures were used, whereas the X-ray energy depended on the tumor location. In case of image-guided IMRT, tumor location was verified with daily cone beam CT with or without the implantation of fiducial markers. A dose-volume histogram (DVH) was generated from a computerized planning system for rigorous evaluation. The prescribed doses for the 2 courses of RT in 36 patients were $30-60$ Gy and 10-60 Gy with a fraction size of $1.8-3 \mathrm{~Gy} ; 2$ of the 36 patients received stereotactic body radiotherapy with a prescribed dose of 35 Gy in 5 fractions.

\section{Follow-up and toxicity definition}

A weekly clinical visit and routine LFT were rigorously conducted during and after the RT course. After the reirradiation course, these patients underwent scheduled LFTs and clinical assessment at least once in 2 weeks in the initial 3 months and monthly follow-up thereafter. For any patient developing $\geq$ grade 3 hepatic toxicities or rapid deterioration of liver function resulting in coagulopathy or encephalopathy, RT was discontinued immediately. When the LFT values deteriorated, the patients were admitted for daily evaluation and supportive care. All toxicities were classified according to the Common Terminology Criteria for Adverse Events (CTCAE), Version 4 [11]. Classic RILD was defined as anicteric hepatomegaly and ascites with more than double the upper limit of the normal level of alkaline phosphate [6]. Moreover, according to the CTCAE criteria, nonclassic RILD was defined as $\geq$ grade 3 hepatic toxicities, with more than 3 times the upper limit of the normal level of blood bilirubin or more than 5 times the upper limit of 
Table 1 Characteristics of all patients

\begin{tabular}{|c|c|c|}
\hline & Patients with RILD & Patients without RILD \\
\hline Variables & $N=13$ & $N=23$ \\
\hline \multicolumn{3}{|l|}{ Gender } \\
\hline Male & 11 & 19 \\
\hline Female & 2 & 4 \\
\hline \multirow[t]{2}{*}{ Median age at reirradiation } & 64 & 71 \\
\hline & (range, 32 to 77 ) & (range, 33 to 89 ) \\
\hline \multicolumn{3}{|l|}{ TNM stage (AJCC 7th edition) } \\
\hline T1NOMO & 2 & 4 \\
\hline T2NOMO & 1 & 7 \\
\hline T3NOMO & 5 & 9 \\
\hline T4N1M0 & 1 & 0 \\
\hline T3N0M1 & 4 & 3 \\
\hline \multicolumn{3}{|l|}{ Previous local treatment } \\
\hline Surgery & 1 & 2 \\
\hline TAE/TACE & 7 & 12 \\
\hline RFA/Cryo & 2 & 5 \\
\hline \multicolumn{3}{|l|}{ Category of previous hepatitis } \\
\hline Hepatitis B infection & 6 & 10 \\
\hline Hepatitis C infection & 2 & 6 \\
\hline non-HBV and non-HCV induced & 5 & 7 \\
\hline CTP scores before the 2 nd RT & $6.3 \pm 1.1$ & $5.2 \pm 0.5$ \\
\hline Interval between 2 courses (months) abutting score for two PTV & $8.1 \pm 8.1$ & $12.6 \pm 12.2$ \\
\hline 0 & 4 & 5 \\
\hline 1 & 2 & 9 \\
\hline 2 & 7 & 9 \\
\hline \multicolumn{3}{|l|}{ 1st RT parameters } \\
\hline Mean prescribed dose $\left(\mathrm{EQD}_{2}, \mathrm{~Gy}\right)$ & $49.5 \pm 8.2$ & $52.5 \pm 5.7$ \\
\hline Mean CTV (cm3) & $392.9 \pm 407.0$ & $345.2 \pm 565.0$ \\
\hline Mean PTV (cm3) & $257.9 \pm 207.8$ & $314.4 \pm 480.7$ \\
\hline Mean normal liver dose $\left(E D_{2}, G y\right)$ & $20.8 \pm 11.3$ & $20.1 \pm 8.2$ \\
\hline Mean normal liver volume (cm3) & $1255.5 \pm 569.4$ & $926.5 \pm 242.4$ \\
\hline \multicolumn{3}{|l|}{ 2nd RT parameters } \\
\hline Mean prescribed dose $\left(\mathrm{EQD}_{2}, \mathrm{~Gy}\right)$ & $32.9 \pm 14.5$ & $40.6 \pm 12.8$ \\
\hline Mean CTV (cm3) & $139.1 \pm 150.2$ & $211.3 \pm 343.1$ \\
\hline Mean PTV (cm3) & $257.9 \pm 207.8$ & $314.4 \pm 480.7$ \\
\hline Mean normal liver dose $\left(E Q D_{2}, G y\right)$ & $12.3 \pm 6.6$ & $10.2 \pm 5.5$ \\
\hline Mean normal liver volume (cm3) & $1173.4 \pm 622.2$ & $992.5 \pm 196.1$ \\
\hline Mean cumulative prescribed dose (EQD $\left.{ }_{2}, G y\right)$ & $80.9 \pm 17.8$ & $94.4 \pm 13.9$ \\
\hline Mean cumulative normal liver dose (EQD, Gy) & $32.5 \pm 15.1$ & $30.5 \pm 9.6$ \\
\hline
\end{tabular}

Abbreviation: RILD radiation-induced liver disease, TNM stage was evaluated before reirradiation; TAE transarterial embolization, TACE transarterial chemo-embolization, RFA radiofrequency ablation, Cryo cryotherapy, $R T$ radiotherapy, CTV clinical tumor volume, PTV planning target volume, EQD2 biologically equivalent doses calculated in $2 \mathrm{~Gy}$

Note: abutting score: if reirradiation applied to a PTV separated from the previous PTV because the tumors were located at different segments, this situation was defined as out-field reirradiation (score $=0$ ). For patients receiving reirradiation at the same or adjacent segment, the 2 nd PTVs might be partially included in the previous PTV (score 1), or completely included (score 2) 
the normal levels of ALT or AST [12]. Lethal RILD was defined as death directly caused by RT-related progressive hepatic failure before or without evidence of tumor progression.

In addition, we examined the impact of location of the two PTVs on the effect of RILD. Irradiation applied to recurrent tumors situated inside the previous PTV was defined as in-field reirradiation. Conversely, irradiation applied to a PTV separated from the previous PTV because the tumors were located at different segments was defined as out-field reirradiation $(N=9)$. For patients receiving reirradiation at the same or adjacent segment $(N=27)$, we proposed an abutting score for classify the location of two PTVs when assessing the impact of volume effect, as described in Table 1.

\section{Statistical analyses}

The primary analysis was identifying the clinical parameters that could predict RILD and lethal RILD. The secondary analysis was identifying prognostic factors associated with survival after reirradiation. Logistic regression analysis was used to identify the predictors of RILD. Variables with a statistical significance in the univariate analysis were included in the multivariate analysis. Overall survival (OS) was defined as the time from the commencement of the second course of RT to the date of death from any cause or the final follow-up. Survival curves were calculated using the Kaplan-Meier method and log-rank test. Cox regression was performed to explore the prognostic factors for OS. The variables analyzed included age, the pretreatment LFT values (CTP score, albumin, total bilirubin, ascites), PTV, normal liver dose of the reirradiation, cumulative normal liver dose, duration, location of the two PTVs, and the presence of portal vein tumor thrombosis (PVTT). A 2-sided $p$ value of $<0.01$ was considered statistically significant. All statistical analyses were performed using SPSS 13.0 for Windows (SPSS Inc., Chicago, IL, USA).

\section{Results}

\section{General outcomes}

The mean follow-up duration after reirradiation was 17 months (range, 3 to 74 months). Thirteen patients (36 \%) developed RILD within 3 months after the initiation of reirradiation. All events were categorized as nonclassic RILD according to their clinical manifestation. Of the 13 patients, 2 could not complete the allocated RT regimen because of hepatic toxicities, and the prescribed doses were 20 Gy in 10 fractions and 21.6 Gy in 12 fractions. There was no evidence to show the continuous deterioration of liver function after the 1st course of RT. Although the previous RT might lead to a subclinical change of liver function, the RILD was mainly attributed to the consequence of reirradiation.

The details of both courses of RT are summarized in the Appendix. We normalized the RT dose by using biologically equivalent doses calculated in 2 Gy as the $\alpha / \beta$ ratio of 15 for $\mathrm{HCC}$ [13] and 8 for the adjacent hepatic tissues [10]. In brief, the mean irradiation doses of the second course of RT for the hepatic tumor and normal liver tissue were $37.52 \mathrm{~Gy}_{15}$ (range, 10$58.68 \mathrm{~Gy}_{15}$ ) and $10.83 \mathrm{~Gy}_{8}$ (range, 2.5-23.97 $\mathrm{Gy}_{8}$ ), respectively. Moreover, the mean cumulative doses to the hepatic tumor and normal liver tissue were 87.70 $\mathrm{Gy}_{15}$ (range, 60.47-117.37 $\mathrm{Gy}_{15}$ ) and $31.11 \mathrm{~Gy}_{8}$ (range, 9.15-62.84 Gy $\mathrm{Gy}_{8}$ ), respectively. The median interval between the 2 courses of RT was 11.0 months (range, 1-47 months).

Among the 13 patients with RILD, 4 had transient grade 3 hepatic toxicity, and the LFT values eventually returned to the normal range within 1 month after the completion of RT. Nine patients had lethal RILD without evidence of intrahepatic tumor progression; 8 patients died of fulminant hepatic failure within 3 months, and the remaining patient died of progressive liver failure 5 months after reirradiation. Furthermore, 2 patients did not complete the prescribed RT course because of a rapid deterioration of hepatic function during treatment, which LFT showed more than 5 times the upper limit of the normal levels of ALT or AST or more than 3 times the upper limit of the normal level of blood bilirubin. In addition to the 13 patients with RILD, 2 patients died of massive bleeding caused by esophageal and gastric varices within 6 months after reirradiation, which was a fatal complication of portal hypertension and classified as nonRILD related death. The other treatment-related adverse events such as RT-induced gastrointestinal bleeding, angiocholitis, and biliary stricture were not observed in this cohort.

\section{Risk factors for RILD and lethal RILD}

To investigate the risk factors for RILD, clinical data, including the pretreatment LFT values, CTP score, and RT parameters before both courses of RT, were analyzed. As shown in Table 2, several pretreatment LFT values and clinical parameters were associated with RILD in univariate analysis. Multivariate analysis revealed that a pretreatment CTP score $\geq 6$ was a predictor of RILD [odds ratio (OR): 15.83, $95 \%$ confidence interval $(\mathrm{CI}): 2.95-85.08, p=0.001]$. The results of univariate and multivariate analyses for lethal RILD are summarized in Table 3. A preirradiation CTP score $\geq 6$ was the only factor associated with lethal RILD [OR: 72.56, $95 \%$ CI: 3.650-1442.252, $p=0.005$ ]. No statistical differences were observed among all 
Table 2 Risk factors associated with RILD after reirradiation

\begin{tabular}{|c|c|c|c|c|c|c|}
\hline \multirow[t]{2}{*}{ Variables } & \multirow[t]{2}{*}{ RILD (+) } & \multirow[t]{2}{*}{ RILD (-) } & \multirow{2}{*}{$\begin{array}{l}\text { Univariate } \\
p \text { value }\end{array}$} & \multicolumn{3}{|c|}{ Multivariate } \\
\hline & & & & $p$ value & OR & $95 \% \mathrm{Cl}$ \\
\hline Total number & 13 & 23 & & & & \\
\hline \multicolumn{7}{|l|}{ Liver function before 2 nd RT } \\
\hline Total bilirubin $\geqq 2.0 \mathrm{mg} / \mathrm{dL}$ & 4 & 0 & 0.016 & & & \\
\hline Albumin $\leqq 3.5 \mathrm{~g} / \mathrm{dL}$ & 8 & 4 & 0.007 & & & \\
\hline Presence of ascites & 3 & 1 & 0.086 & & & \\
\hline INR $\geqq 1.71$ & 0 & 0 & & & & \\
\hline CTP score $\geqq 6$ & 10 & 4 & $<0.0001$ & 0.001 & 15.833 & $2.947 \sim 85.075$ \\
\hline Presence of PVTT & 8 & 5 & 0.017 & & & \\
\hline AST/ALT $\geqq 3 x$ of upper normal limit & 5 & 1 & 0.088 & & & \\
\hline \multicolumn{7}{|l|}{ Hepatitis } \\
\hline Hepatitis B infection & 6 & 10 & 0.877 & & & \\
\hline Hepatitis C infection & 2 & 6 & 0.458 & & & \\
\hline \multicolumn{7}{|l|}{ RT parameters of 2nd RT } \\
\hline Mean CTV $\left(\mathrm{cm}^{3}\right)$ & $158.8 \pm 157.9$ & $204.2 \pm 348.7$ & 0.740 & & & \\
\hline Mean PTV $\left(\mathrm{cm}^{3}\right)$ & $257.9 \pm 207.8$ & $314.4 \pm 480.7$ & 0.260 & & & \\
\hline Mean normal liver dose $\left(\mathrm{EQD}_{2}, \mathrm{~Gy}_{8}\right)$ & $12.0 \pm 6.8$ & $10.2 \pm 4.8$ & 0.726 & & & \\
\hline Mean normal liver volume $\left(\mathrm{cm}^{3}\right)$ & $1173.4 \pm 622.2$ & $992.5 \pm 196.1$ & 0.332 & & & \\
\hline Interval between 2 courses (month) & $8.1 \pm 8.1$ & $12.6 \pm 12.3$ & 0.247 & & & \\
\hline Mean cumulative normal liver dose $\left(\mathrm{EQD}_{2}, \mathrm{~Gy}_{8}\right)$ & $32.0 \pm 15.0$ & $30.6 \pm 9.7$ & 0.233 & & & \\
\hline Mean cumulative prescribed dose $\left(\mathrm{EQD}_{2}, \mathrm{~Gy}_{15}\right)$ & $82.8 \pm 15.9$ & $90.6 \pm 15.4$ & 0.065 & & & \\
\hline Abutting score for two PTV & $1.3 \pm 0.9$ & $1.1 \pm 0.7$ & 0.548 & & & \\
\hline
\end{tabular}

Abbreviation: RILD radiation-induced liver disease, OR odds ratio, Cl confidence interval, CTP Child-Turcotte-Pugh, PVTT portal vein tumor thrombosis, AST aspartate aminotransferase, ALT alanine aminotransferase, CTV clinical tumor volume, NS no significance, EQD2 biologically equivalent doses calculated in 2Gy

dosimetric parameters between patients with and those without RILD. In addition, the abutting score for the two PTVs and the treatment interval did not affect RILD. Furthermore, the pretreatment LFT before the 1st course of RT and normal liver dose or volume of the first RT course did not affect the occurrence of RILD after reirradiation (Appendix).

\section{Prognostic factors for OS}

At the time of this analysis, 32 patients died of disease progression with or without hepatic failure. Patients with RILD exhibited a shorter OS than did those without RILD, with the median survival being 5.7 and 29.0 months, respectively $(p<0.001$; Fig. 1$)$. The median survival decreased to 3.8 months in patients with lethal RILD. In multivariate analysis, the presence of PVTT [hazard ratio (HR): 12.41, 95 \% CI: 2.05-12.41, $p<0.001$ ) and a CTP score $\geq 6$ (HR: 8.79, $95 \%$ CI: $1.48-6.74, p=0.003)$ before reirradiation were prognostic factors. As depicted in Fig. 1, the median OS of patients with and without PVTT was 5 months and 22 months, respectively $(p<0.001)$, whereas that of patients with CTP scores of $\geq 6$ and 5 was 4 months and 18 months $(p<0.001)$, respectively. No statistical difference was observed regarding OS for other clinical parameters, such as age, the irradiation interval, and the dosimetric parameters.

\section{Discussion}

In advanced HCC, RT is assumed to delay the time to tumor progression and resolves vascular damage caused by PVTT. However, the benefits are reduced by the increased risk of RT-related hepatic toxicities. Several studies have reported clinical parameters associated with RILD [14-18]; however, currently, no guidelines exist regarding hepatic tolerance or risk factors for RILD after reirradiation. This study is the first to reveal that a CTP score $\geq 6$ is associated with an increased risk of RILD and predicts the occurrence of lethal RLID. In particular, the estimated OR was more than 10-fold higher than that of patients with a CTP score of 5. In addition, a high mortality rate can be anticipated in patients with a CTP score $\geq 6$. In our study, patients with a pretreatment CTP score $\geq 6$ had a median OS of less than 6 months; 
Table 3 Risk factors associated with lethal RILD after reirradiation

\begin{tabular}{|c|c|c|c|c|c|c|}
\hline \multirow[t]{2}{*}{ Variables } & \multirow[t]{2}{*}{ Lethal RILD (+) } & \multirow[t]{2}{*}{ Lethal RILD (-) } & \multirow{2}{*}{$\begin{array}{l}\text { Univariate } \\
p \text { value }\end{array}$} & \multicolumn{3}{|c|}{ Multivariate } \\
\hline & & & & $p$ value & $\mathrm{OR}$ & $95 \% \mathrm{Cl}$ \\
\hline Total number & 9 & 27 & & & & \\
\hline \multicolumn{7}{|l|}{ Liver function before 2 nd RT } \\
\hline Total bilirubin $\geqq 2.0 \mathrm{mg} / \mathrm{dL}$ & 4 & 0 & 0.002 & & & \\
\hline Albumin $\leqq 3.5 \mathrm{~g} / \mathrm{dL}$ & 6 & 6 & 0.001 & & & \\
\hline Presence of ascites & 3 & 1 & 0.014 & & & \\
\hline $\mid N R \geqq 1.71$ & 0 & 0 & & & & \\
\hline CTP score $\geqq 6$ & 8 & 6 & $<0.001$ & 0.005 & 72.5554 & $3.650 \sim 1442.252$ \\
\hline Presence of PVTT & 7 & 6 & 0.003 & & & \\
\hline AST/ALT $\geqq 3 x$ of upper normal limit & 4 & 2 & 0.121 & & & \\
\hline \multicolumn{7}{|l|}{ Hepatitis } \\
\hline Hepatitis B infection & 5 & 11 & 0.439 & & & \\
\hline Hepatitis C infection & 1 & 7 & 0.355 & & & \\
\hline \multicolumn{7}{|l|}{ RT parameters of 2 nd RT } \\
\hline Mean CTV $\left(\mathrm{cm}^{3}\right)$ & $195.2 \pm 166.9$ & $186.4 \pm 331.7$ & 0.806 & & & \\
\hline Mean PTV $\left(\mathrm{cm}^{3}\right)$ & $315.5 \pm 224.5$ & $285.7 \pm 447.7$ & 0.890 & & & \\
\hline Mean normal liver dose $\left(\mathrm{EQD}_{2}, \mathrm{~Gy}_{8}\right)$ & $9.7 \pm 6.8$ & $11.3 \pm 5.2$ & 0.019 & & & \\
\hline Mean normal liver volume $\left(\mathrm{cm}^{3}\right)$ & $1305.8 \pm 687.6$ & $975.2 \pm 210.5$ & 0.078 & & & \\
\hline Interval between 2 courses (month) & $6.0 \pm 8.5$ & $12.7 \pm 11.4$ & 0.119 & & & \\
\hline Mean cumulative normal liver dose $\left(\mathrm{EQD}_{2}, \mathrm{~Gy}_{8}\right)$ & $27.9 \pm 12.5$ & $32.2 \pm 11.5$ & 0.221 & & & \\
\hline Mean cumulative prescribed dose $\left(\mathrm{EQD}_{2}, \mathrm{~Gy}_{15}\right)$ & $75.0 \pm 12.7$ & $92.1 \pm 14.7$ & 0.005 & & & \\
\hline Abutting score for two PTV & $1.4 \pm 0.8$ & $1.1 \pm 0.8$ & 0.824 & & & \\
\hline
\end{tabular}

Abbreviation as Table 2

therefore, tailored selection is essential for patients requiring a second course of $\mathrm{RT}$.

This study analyzed several pretreatment LFT parameters and concluded that the CTP score, which is widely used for the clinical assessment of liver cirrhosis, was a stronger predictor of hepatic toxicities than the other parameters. In addition, our study revealed that the duration between the 2 courses of RT and the dosimetric parameters did not affect the risk of hepatic toxicities, implying that residual liver function still plays a major role in determining the tolerance to reirradiation. This finding is consistent with the findings of several studies $[3,12,14-17,19,20]$. Yoon reported no correlation between RILD and DVH parameters, including the total

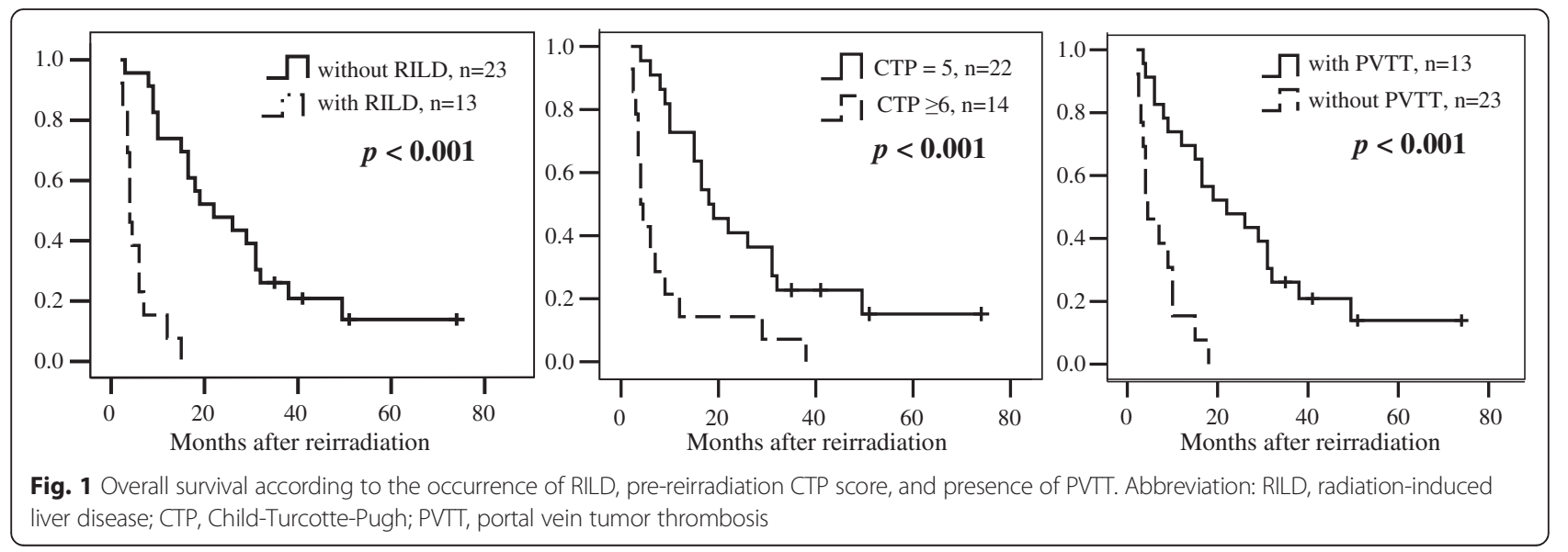


liver volume, mean liver dose, V30, normal tissue complication probability (NTCP), and equivalent uniform dose [15]. Cheng et al. proposed that patients with HCC having HBV infection and a Child-Pugh class of B have a high risk of RILD [14]. Liang et al. suggested that the severity of hepatic cirrhosis is a major prognostic factor for RILD. In addition, some studies have suggested that the indocyanine green retention rate at 15 min (ICGR15) is an accurate tool for predicting $\operatorname{RILD}[15,16]$. Lee et al. indicated that the CTP score is a more reliable predictor of RILD than is the ICG-R15 [21]. By contrast, Pan et al. conducted quantitative analyses of normal tissue in a clinic and suggested applying stricter dose constraints to RT for HCC than for metastatic hepatic tumors, namely mean normal liver doses of less than 28 Gy for HCC and less than $32 \mathrm{~Gy}$ for liver metastases for therapeutic partial liver RT (in 2-Gy fractions) [22]. To reach a widely accepted consensus, clinical trials analyzing comprehensive DVH data, clinical parameters, and the HBV titer should be conducted to determine patients with a high risk of RILD after reirradiation.

Although our study showed no impact of location of the two PTVs on RILD, the effect of the dose distribution pattern on outcomes requires further investigation. If recurrent tumors are situated inside the previous PTV, the surrounding hepatic tissues receive a summation of doses from both courses of RT. Dawson et al. proposed that a partial liver can tolerate more than 100 Gy if the volume is smaller than $25 \%$ [5]. By contrast, the cumulative low dose volume is larger when recurrent tumors are far from the previous PVTT. According to a similar NTCP model [5], two-thirds of the total liver volume was assumed to tolerate doses of less than $48 \mathrm{~Gy}$, whereas the total liver volume can tolerate doses of less than 32 Gy. In this study, we compared the cumulative dose to the normal liver rather than the detailed DVH profile because of the difficulty of summing DVHs from both courses of RT. Therefore, comprehensive DVH data should be retrieved for future analysis.

PVTT is an established prognostic factor for survival in patients with HCC receiving hepatic RT [3]. Our study revealed that PVTT had a significant effect on OS after reirradiation. Therefore, in patients with PVTT, the risk of shortened survival should be considered before reirradiation. In a retrospective study of a cohort of 46 patients with HCC receiving RT [3], Furuse et al. reported that PVTT exerted substantial effects on acute adverse hepatic toxicities, survival, and tumor response. They also confirmed that acute hepatic toxicities, including hyperbilirubinemia and hypoalbuminemia, were critical factors affecting survival. Therefore, in the future, we plan to clarify the association between the grade of the tumor thrombus and adverse hepatic events or treatment outcomes.

Our study results should be interpreted with caution. First, the results of this retrospective study with a limited sample size from 2 institutes should be prospectively validated using different irradiation schemes and patient populations. In addition, complete parameters of liver function such as gamma glutamyl transferase or virus titers were imperative to monitor the hepatic damage. Furthermore, the place of SBRT was still unclear in this study because of different biological dose effect compared to conventional radiotherapy. A prospective trial using SBRT for recurrent hepatic tumor is essential to maximize the therapeutic ratio. Finally, the conclusion can be strengthened by collecting and comparing more comprehensive DVH parameters. Thus, a modified NTCP model can be designed for obtaining an accurate score of the risk of RILD following reirradiation. Nonetheless, our study has implications for decision-making regarding initiating the second course of hepatic RT or stratifying patients in clinical trials. With more careful and thorough evaluation, this study can help radiation oncologists more meticulously re-treat patients with HCC, and survival benefits can be expected in patients who do not develop RILD. Early prediction of potential hepatic toxicities would enable administering individualized therapy to patients requiring a second course of hepatic RT.

\section{Conclusion}

Although this study included low number of patients into the analysis, a pretreatment CTP score $\geq 6$ is associated with an increased risk of RILD and lethal RILD in patients requiring a second course of hepatic RT. Patients with RILD have a high mortality rate, implying that residual liver function plays a crucial role in determining the tolerance to reirradiation and survival after treatment. A multicenter prospective study is required to examine additional comprehensive risk factors for RILD.

\section{Additional information - Ethics approval}

This retrospective study was approved by Taipei Medical University - Joint Institutional Review Board with approval no. N201508033.

\section{Consent for publication}

Not applicable.

\section{Availability of data and materials}

Not applicable. 


\section{Appendix}

Table 4 Pretreatment liver function before the 1st course of RT and the association with RILD after reirradiation

\begin{tabular}{|c|c|c|c|}
\hline Variables & RILD (+) & RILD (-) & $\begin{array}{l}\text { Univariate } \\
p \text { value }\end{array}$ \\
\hline Total number & 13 & 23 & \\
\hline \multicolumn{4}{|l|}{ Liver function before 1st RT } \\
\hline Total bilirubin $\geqq 2.0 \mathrm{mg} / \mathrm{dL}$ & 0 & 0 & \\
\hline Albumin $\leqq 3.5 \mathrm{~g} / \mathrm{dL}$ & 5 & 3 & 0.54 \\
\hline Ascites & 0 & 0 & \\
\hline INR $\geqq 1.71$ & 0 & 0 & \\
\hline CTP score $\geqq 6$ & 4 & 2 & 0.77 \\
\hline AST/ALT $\geqq 3 x$ of upper normal limit & 3 & 2 & 0.38 \\
\hline Presence of PVTT & 5 & 3 & 0.75 \\
\hline \multicolumn{4}{|l|}{ RT parameters } \\
\hline Mean PTV of the 1st RT $\left(\mathrm{cm}^{3}\right)$ & $605.7 \pm 551.5$ & $410.6 \pm 667.6$ & 0.41 \\
\hline Mean normal liver dose of 1st RT $\left(\mathrm{EQD}_{2}, \mathrm{~Gy}_{8}\right)$ & $20.8 \pm 12.0$ & $21.3 \pm 8.7$ & 0.87 \\
\hline Mean normal liver volume of 1st RT $\left(\mathrm{cm}^{3}\right)$ & $1255.5 \pm 592.4$ & $1055.3 \pm 274.3$ & 0.17 \\
\hline
\end{tabular}

Abbreviation: RILD radiation-induced liver disease, OR odds ratio, Cl confidence interval, CTP Child-Turcotte-Pugh, PVTT portal vein tumor thrombosis, AST aspartate aminotransferase, $A L T$ alanine aminotransferase, $C T V$ clinical tumor volume, NS no significance, EQD2 biologically equivalent doses calculated in $2 \mathrm{~Gy}$

\section{Abbreviation}

ALT, alanine aminotransferase; AST, aspartate aminotransferase; $C T$, computed tomography; CTCAE, Common Terminology Criteria for Adverse Events; CTP, Child-Turcotte-Pugh; CTV, clinical target volume; DVH, dose-volume histogram; GTV, gross tumor volume; HBV, hepatitis B virus; $\mathrm{HCC}$, hepatocellular carcinoma; ICG-R15, indocyanine green retention rate at $15 \mathrm{~min}$; LFT, liver function test; NTCP, normal tissue complication probability; OS, overall survival; PTV, planning target volume; PVIT, portal vein tumor thrombosis; RILD, radiation-induced liver disease; RT, radiotherapy

\section{Competing interests}

The authors declare that they have no competing interests.

\section{Authors' contributions}

YH co-conceived the study, gathered the patients' data, participated in the statistical analysis and interpretation and drafted the manuscript. SC performed the statistical analysis and interpretation, participated in study design and helped to draft the manuscript. CF, LT, and CK assisted in acquisition of patients' data and study design. JC conceived of the study, participated in its design and coordination. All authors read and approved the final manuscript.

\section{Acknowledgment}

We thank the medical physicist Dong-Ho Chen for helping in organizing dose-volume histogram parameters from the radiotherapy records.

\section{Funding}

This retrospective study received no any funding.

\section{Author details}

'Department of Radiation Oncology, Taipei Medical University Hospital, 252, Wu Hsing St., Taipei 110, Taiwan. ${ }^{2}$ Department of Radiation Oncology, China Medical University Hospital, Taichung, Taiwan. ${ }^{3}$ Department of Radiology, School of Medicine, College of Medicine, Taipei Medical University, Taipei, Taiwan. ${ }^{4}$ Department of Radiation Oncology, Saint Mary's Hospital, Luodong, Taiwan.
Received: 5 February 2016 Accepted: 19 June 2016

Published online: 02 July 2016

References

1. Dawson LA, Guha C. Hepatocellular carcinoma: radiation therapy. Cancer J. 2008:14:111-6.

2. Klein J, Dawson LA. Hepatocellular carcinoma radiation therapy: review of evidence and future opportunities. Int J Radiat Oncol Biol Phys. 2012 87:22-32.

3. Furuse J, Ishi $\mathrm{H}$, Nagase $\mathrm{M}$, et al. Adverse hepatic events caused by radiotherapy for advanced hepatocellualr carcinoma. J Gastroenterol Hepatol. 2005:20:1512-8.

4. Dawson $L A$, Haken R. Partial volume tolerance of the liver to radiation. Semin Radiat Oncol. 2005:15:279-83.

5. Dawson LA, Normolle D, Balter J, et al. Analysis of radiation-induced liver disease using the Lyman NTCP models. Int J Radiat Oncol Biol Phys. 2002; 53:810-21.

6. Guha C, Kavanagh BD. Hepatic radiation toxicity: avoidance and amelioration. Semin Radiat Oncol. 2011:21:256-63.

7. Son $\mathrm{SH}$, Jang $\mathrm{HS}$, Lee $\mathrm{H}$, et al. Determination of the $\alpha / \beta$ ration for the normal liver on the basis of radiation-induced hepatic toxicities in patients with hepatocellular carcinoma. Radiat Oncol. 2013;8:61.

8. Dawson LA. Overview: Where does radiation therapy fit in the spectrum of liver cancer local-regional therapies? Semin Radiat Oncol. 2011;21:241-6.

9. Cholongitas E, Papatheodoridis GV, Vangeli M, et al. Systemic review: the model for end-stage liver disease - should it replace child-Pugh's classification for assessing prognosis in cirrhosis? Aliment Pharmacol Ther. 2005;22:1079-89.

10. Marks $L B$, Yorke ED, Jackson $A$, et al. Use of normal tissue complication probability models in the clinic. Int I Radiat Oncol Biol Phys. 2010;76:S10-19.

11. Common Terminology Criteria for Adverse Events (CTCAE) v4.0. http://ctep. cancer.gov/protocolDevelopment/electronic_applications/ctc.htm

12. Cheng JC, Wu J-K, Huang C-M, et al. Radiation-induced liver disease after radiotherapy for hepatocellular carcinoma: clinical manifestation and dosimetric description. Int J Radiat Oncol Biol Phys. 2002;53:156-62.

13. Hennequin C, Quero L, Rivera S. Radiosensitivity of hepatocellular carcinoma. Cancer Radiother. 2011;15(1):39-42. 
14. Cheng JC, Wu J-K, Lee PC, et al. Biologic susceptibility of hepatocellular carcinoma patients treated with radiotherapy to radiation-induced liver disease. Int J Radiat Oncol Biol Phys. 2004;60:1502-9.

15. Yoon HI, Koon WS, Lee IJ, et al. The significance of ICG-R15 in predicting hepatic toxicity in patients receiving radiotherapy for hepatocellular carcinoma. Liver Int. 2012;32(7):1165-71.

16. Stenmark MH, Cao $\mathrm{Y}$, Wang $\mathrm{H}$, et al. Estimating functional liver reserve following hepatic irradiation: adaptive normal tissue response models. Radiother Oncol. 2014;111(3):418-23.

17. Llovet JM, Ducreux M, Lencioni R, et al. European association for the study of the liver EASL-EORTC clinical practice guidelines: management of hepatocellular carcinoma. J Hepatol. 2012;56:908-43.

18. Que JY, Lin LC, Lin KL, et al. The efficacy of stereotactic body radiation therapy on huge hepatocellular carcinoma unsuitable for other local modalities. Radiat Oncol. 2014:9:120

19. Liang S-X, Zhu X-D, Xu Z-Y, et al. Radiation-induced liver disease in threedimensional conformal radiation therapy for primary liver carcinoma: the risk factors and hepatic radiation tolerance. Int J Radiat Oncol Biol Phys. 2006;65:426-34

20. Xu Z-Y, Liang S-X, Zhu J, et al. Prediction of radiation-induced liver disease by Lyman normal tissue complication probability model in threedimensional conformal radiation therapy for primary liver carcinoma. Int J Radiat Oncol Biol Phys. 2006;65:189-95.

21. Lee IJ, Seong J, Shim SJ, et al. Radiotherapeutic parameters predictive of liver complications induced by liver tumor radiotherapy. Int J Radiat Oncol Biol Phys. 2009:73:154-8

22. Pan CC, Kavanagh BD, Dawson L, et al. Radiation-associated liver injury. Int J Radiat Oncol Biol Phys. 2010;76:S94-100.

\section{Submit your next manuscript to BioMed Central and we will help you at every step:}

- We accept pre-submission inquiries

- Our selector tool helps you to find the most relevant journal

- We provide round the clock customer support

- Convenient online submission

- Thorough peer review

- Inclusion in PubMed and all major indexing services

- Maximum visibility for your research

Submit your manuscript at www.biomedcentral.com/submit

) Biomed Central 